\title{
Aflatoxins M1 and M2 in the milk of donkeys fed with naturally contaminated diet
}

\author{
B. Tozzi $^{1} \cdot$ G.B. Liponi ${ }^{1} \cdot$ V. Meucci $^{1} \cdot$ L. Casini $^{1}$ • \\ C. Dall'Asta ${ }^{2} \cdot$ L. Intorre ${ }^{1} \cdot$ D. Gatta ${ }^{1}$
}

Received: 22 September 2015 / Revised: 4 March 2016 / Accepted: 7 March 2016 /

Published online: 13 April 2016

C) INRA and Springer-Verlag France 2016

\begin{abstract}
For its nutritional composition, donkey milk is an excellent alternative to breast milk for infants suffering from cow's milk allergies. Even in donkeys, a passage of aflatoxin from contaminated feed to milk could occur, as reported by many authors in other dairy species, but there are no studies on this topic. This work was aimed at studying the excretion of aflatoxin M1 (AFM1) and M2 (AFM2) in milk after feeding trials with contaminated feed. Six donkeys, at the end of lactation, received a diet with naturally contaminated corn containing 202 and $11 \mu \mathrm{g} \cdot \mathrm{kg}^{-1}$ of aflatoxin B1 (AFB1) and aflatoxin B2 (AFB2), respectively. Individual milk samples were analyzed for AFM1 and AFM2 for 15 days after the contaminated feed administration. Amounts of AFM1 and AFM2 were detected in the milk. The steady state condition was reached after 6 days. No AFM1 or AFM2 were detected in milk after $28 \mathrm{~h}$ from the last contaminated feed administration. The carryover from AFB1 to AFM1 and from AFB2 to AFM2 was found to be 0.02 and $0.31 \%$, respectively. The results obtained in this study are thus a further step toward understanding the possible carryover of aflatoxin in donkey milk.
\end{abstract}

Keywords Aflatoxins·Donkey·Carryover·Milk

\section{Introduction}

Donkey milk, with its natural and healthful properties, is unique as an optimal substitute for infants allergic to cows' milk and for the elderly. The presence of aflatoxins in animal products poses a serious risk to consumers' health. Even in donkey

B. Tozzi

btozzi@vet.unipi.it

1 Dipartimento di Scienze Veterinarie, Università di Pisa, Viale delle Piagge 2, 56124 Pisa, Italy

2 Dipartimento di Chimica Organica e Industriale, Università degli Studi di Parma, Viale G.P. Usberti 17/a, 43124 Parma, Italy 
milk, as in the milk of other species, aflatoxins from feed may be present. However, no studies have been carried out on donkeys to investigate the carryover of aflatoxin from feed to milk and to evaluate the associated risk.

Aflatoxins (AFs) are one of the best known and widely investigated groups of mycotoxins which can be found as contaminants in food commodities worldwide. AFs are mainly produced by the Aspergillus species in agricultural products such as grains, oil seeds, beans, nuts, and dried fruits (Ardic et al. 2008; Decastelli et al. 2007), as well as in hays and ensiled forages. Besides their acute toxicity, mainly targeted at the liver, aflatoxins are well-known carcinogenic, teratogenic, and mutagenic compounds. In particular, aflatoxin B1 (AFB1), B2 (AFB2), G1, and G2, together with the main oxidative metabolite aflatoxin M1, have been classified as Group I human carcinogens by the IARC (IARC 2002).

After ingestion, AFB1 and AFB2 are metabolized by the liver to their hydroxylated metabolites M1 (AFM1) and M2 (AFM2), which can be excreted in urine, feces, transferred to milk and, to a lesser extent, to meat (Fink-Gremmels 2008). Although the AFB1 to AFM1 conversion ratio in milk is rather low (1-3\%), milk contamination is of concern due to the key role played by milk and dairy products in children's diet (López et al. 2003; Herzallah 2009). For this reason, the European Union has set $0.05 \mu \mathrm{g} \cdot \mathrm{kg}^{-1}$ as the limit for AFM1 in milk and $0.025 \mu \mathrm{g} \cdot \mathrm{kg}^{-1}$ for AFM1 in infant formulas and follow-on formulas, including infant milk and follow-on milk (Commission Regulation (EC) N. 1881/2006). Additionally, the Commission Regulation (EU) N. 574/2011 has set the limit for AFB1 at $20 \mu \mathrm{g} . \mathrm{kg}^{-1}$ for feed materials, $10 \mu \mathrm{g} \cdot \mathrm{kg}^{-1}$ for complementary and complete feed, and $5 \mu \mathrm{g} \cdot \mathrm{kg}^{-1}$ for compound feed for dairy cattle and calves, dairy sheep and lambs, dairy goats and kids, piglets and young poultry animals. No limits are set for AFM2 in milk, while maximum levels have been established in feed for the total amount of aflatoxins.

Donkey milk is quite marginal and is mostly produced for cosmetic use or as substitute to human milk in European small-scale farms that have chosen diversified production (Jirillo et al. 2010). Donkeys, together with horses and yaks, produce less than $0.1 \%$ of milk from all species including cattle, but no world-specific statistics are available (Faye and Konuspayeva 2012). Nevertheless, nowadays, the consumers' interest in donkey milk is increasing, and this product is gaining importance and international acceptance. In the matrix Boston Consulting Group (BCG), this milk is among the products "Question Mark," characterized by low market share and by a high potential of development. Actually, donkey's milk is a niche business with high commercial value (Moruzzo and Rossignoli 2013).

Donkey feed is based on forage, thus to increase donkey milk production, it is necessary to supplement the diet with energy sources such as cereals which, in particular climatic condition, are the main target of aflatoxin B1 and B2.

While for lactating ruminants, such as cows, sheep, and goats, a large literature exists; in the case of lactating donkeys very, little is known on the occurrence and the transfer of AFB1 and AFB2 as AFM1 and AFM2 from feed into milk.

Kos et al. (2013) investigated the occurrence of AFM1 in milk of different species in Serbia. In particular, the authors detected AFM1 in the milk produced by three donkeys, but no information was available about the AFB1 amount ingested by the animals. Very recently, Bilandzic et al. (2014) analyzed 14 samples of donkey milk from Serbia, finding an average contamination of $4.77 \pm 1.33 \mathrm{ng} . \mathrm{L}^{-1}$. As milk samples 
were taken in the summer and AFM1 levels in donkeys' milk were lower than in cows' milk, the authors speculated about the availability of pastures and forage for animals during summer.

Because of the lack of knowledge on the carryover of aflatoxins B in donkey milk, an experiment was conducted to study the relationship between AFB1 and AFB2 intake and AFM1 and AFM2 excretion in donkeys fed naturally contaminated corn.

\section{Material and methods}

Six Romagnola breed donkeys at the end of lactation (average 218 days in milking) were used. Daily milk yield and body weight (BW) averaged to $1.9 \pm 0.3 \mathrm{~L}$ and 259.7 $\pm 6.62 \mathrm{~kg}$ (mean $\pm \mathrm{SD}$ ) respectively.

Before the trial started and during the experimental period, the health of the animals was monitored continuously. Body weights of donkeys were measured before and at the end of the trial.

The experiment was conducted in accordance with the guidelines of the EC Council Directive (86/609/EEC).

Each animal was fed $8 \mathrm{~kg}$ of hay, $0.5 \mathrm{~kg}$ of mixed flakes, and $1 \mathrm{~kg}$ of corn for 25 days. The hay and mixed flakes administered during the trial were not contaminated by aflatoxins. The corn administered during the adaptation period and the last 3 days of the trial was not contaminated by AFs. After a 10-day adaptation period, until the 22nd day, the corn was replaced with corn naturally contaminated (CNC) by $202 \mu \mathrm{g} . \mathrm{kg}^{-1}$ of AFB1 and $11 \mu \mathrm{g} \cdot \mathrm{kg}^{-1}$ of AFB2. During the experimental period, the CNC was individually administered by a bucket in the morning. The animals were monitored in order to ensure that the CNC was completely ingested.

Individual milk samples were collected at $-24,0,1,4,8,24,32,48,56,80,104$, $128,152,224$, and $296 \mathrm{~h}$ after the first contaminated corn administration. At the $22 \mathrm{nd}$ day, the animals were fed for the last time with $\mathrm{CNC}$ and individual milk samples were collected at $0,4,8,16,20,24,28,32,48$, and $72 \mathrm{~h}$ after the last administration. Milk samples were stored at $-20^{\circ} \mathrm{C}$ until the analyses for AFs had been performed.

\subsection{Chemicals and reagents}

The AFM1 reference standard was provided by Sigma-Aldrich (Milan, Italy). The AFM2 reference standard was provided by Fermentek (Jerusalem, Israel). HPLC grade water and solvents were provided from VWR-PBI (Milan, Italy). The immunoaffinity columns (IAC) IClean C Afla were provided by Tecna Srl (Trieste, Italy). A commercial solution of $\mathrm{AFB}_{1}, \mathrm{AFB}_{2}, \mathrm{AFG}_{1}$, and $\mathrm{AFG}_{2}$ in methanol, $1000 \mathrm{ng} \cdot \mathrm{mL}^{-1}$, was provided by Aflastandard, R-Biopharm, Madrid, Spain. All other reagents were of analytical gradient.

\subsection{Determination of AFM1 and AFM2}

Milk analysis was performed by the HPLC-FLD method according to Meucci et al. (2010), slighty modified. Milk samples were previously skimmed by means of the addition of Celite (Sigma-Aldrich) and centrifugation at $1006 \mathrm{x} \mathrm{g}$ for $10 \mathrm{~min}$. After elimination of the 
fat layer, $50 \mathrm{~mL}$ of milk was filtered through Whatman filter paper (Whatman International, Maidstone, UK) and then passed through IAC at a flow-rate of one to two drops per second. The column was washed with $20 \mathrm{~mL}$ of phosphate-buffered saline (PBS) (one to two drops per second). Elution was performed with $3 \mathrm{~mL}$ of methanol. The eluent was evaporated under stream of nitrogen till it became dry. The residue was redissolved in $250 \mu \mathrm{L}$ of HPLC mobile phase and injected into the HPLC system.

The chromatographic system consisted of a Jasco880 pump and a Jasco821 fluorescence detector (Jasco, Tokyo, Japan). JascoBorwin software was used for data processing. The excitation wavelength and emission wavelength were set at 365 and $435 \mathrm{~nm}$. The reversed-phase column was a Luna C18 ODS2, $3 \mu \mathrm{m},(4.6 \times 150 \mathrm{~mm})$ (Phenomenex, Torrance, CA, USA). The column was kept at room temperature. The HPLC was operated with a mobile phase system consisting acetonitrile-water 23/ $77 \% v / v$ at a flow rate of $1 \mathrm{~mL} \cdot \mathrm{min}^{-1}$.

\subsection{Confirmation of AFM2 occurrence in milk by LC-MS/MS}

The chromatographic analysis for milk samples revealed an additional peak related to AFM1. In consideration of the specificity of the analytical protocol and the possible cross-reaction with immunoaffinity antibodies, the peak was tentatively identified as AFM2.

Confirmation of AFM2 identity in milk has been performed by HPLC-MS/MS according to Biancardi et al. (2013) slighty modified. Milk samples were centrifuged at $1006 \mathrm{x} \mathrm{g}$ for $15 \mathrm{~min}$ and the fat layer discharged. A total of $20 \mathrm{~g}$ of the defatted milk was added to sodium chloride $(2 \mathrm{~g})$ and ethyl acetate $(20 \mathrm{~mL})$. The mixture was stirred for $10 \mathrm{~min}$, and then centrifuged at $447 \mathrm{~g}$ for $5 \mathrm{~min}$. A total of $5 \mathrm{~mL}$ of the organic layer was evaporated under nitrogen flow (at $40{ }^{\circ} \mathrm{C}$ ) and the residue was redissolved in $250 \mu \mathrm{L}$ of formic acid 0.1\%/acetonitrile 90:10 $(v / v)$, vortexed and sonicated for $5 \mathrm{~min}$. After centrifugation at $1006 \mathrm{x} g$ for $10 \mathrm{~min}, 170 \mu \mathrm{L}$ of the solution were transferred in a vial for the LC analysis.

LC-MS/MS analysis was performed with a 6430 Triple Quad MS (Agilent Technologies, Santa Clara, CA, USA) equipped with an electrospray interface and a 1290 Infinity separation module (Agilent Technologies). The separation was achieved on a ZORBAX SB-C18 (Agilent Technologies; $50 \mathrm{~mm}, 2.1 \mathrm{~mm}$ I.D., $1.8 \mathrm{~mm}$ ) column with a precolumn filter $(0.3 \mu \mathrm{m})$. Gradient elution was performed using eluent $\mathrm{A}, 0.1 \%$ formic acid in water, and eluent B, $0.1 \%$ formic acid in acetonitrile. The gradient was set as follows: the first step was a linear gradient from $95 \%$ A to $95 \%$ B in $5 \mathrm{~min}$, followed by a 1-min isocratic step at $95 \% \mathrm{~B}$. The column was reconditioned at $95 \% \mathrm{~A}$ for $1.5 \mathrm{~min}$. The total run time was $7.5 \mathrm{~min}$. The following parameters were set: flow rate $0.4 \mathrm{~mL} . \mathrm{min}^{-1}$, column temperature $40{ }^{\circ} \mathrm{C}$, injection volume $10 \mathrm{~mL}$.

\subsection{Determination of aflatoxins in feed}

Feeds analysis was performed by the HPLC coupled to Kobra cell method according to Hernandez-Martınez and Navarro-Blasco (2010), slighty modified. Feeds $(50 \mathrm{~g})$ were carefully weighed and mixed with $4 \mathrm{~g}$ of sodium chloride and $250 \mathrm{~mL}$ of extracting agent acetonitrile/water $(60: 40, v / v)$ into a blender jar. After crushing and mixing for $2 \mathrm{~min}$ at high speed, the extract was filtered through 
Whatman No. 4 filter paper (Whatman International, Maidstone, UK). A 25-mL volume of filtrate was evaporated (Buchi R-3000 Rotavapor; Büchi Labortechnik $\mathrm{AG}$, Postfach, Switzerland) for $8 \mathrm{~min}$ at a temperature of $30{ }^{\circ} \mathrm{C}$ and a rotation speed of $65 \mathrm{rpm}$. The evaporation residue was transferred into $50-\mathrm{mL}$ volumetric flasks and topped up with $500 \mu \mathrm{L}$ acetonitrile and a solution of PBS. Then, $10 \mathrm{~mL}$ of the reconstituted extract was passed through the immunoaffinity column (Tecna, Trieste, Italy) at a flow rate of $2 \mathrm{~mL} \cdot \mathrm{min}^{-1}$. The column was washed with two aliquots of $10 \mathrm{~mL}$ ultrapure water at a flow rate of $5 \mathrm{~mL} \cdot \mathrm{min}^{-1}$, and the aflatoxins were slowly released from the antibody using $1 \mathrm{~mL}$ methanol and eluted with $1 \mathrm{~mL}$ ultrapure water. Finally, the eluted samples were filtered via a PVDF syringe filter (13 mm, $0.22 \mu \mathrm{m}$; Tecnokroma, Barcelona, Spain), and then collected in vials for HPLC analysis.

The chromatographic system consisted of a Jasco880 pump and a Jasco821 fluorescence detector (Jasco, Tokyo, Japan). JascoBorwin software was used for data processing. A Kobra Cell system (R-Biopharm) was used for postcolumn derivatization. Separation was carried out on a Luna $\mathrm{C}_{18}$ column of $4.6 \times 150 \mathrm{~mm}, 5 \mu \mathrm{m}$ particle size, $100 \AA$ (Phenomenex, Torrance, CA, USA) protected by a precolumn (Phenomenex): precolumn holder, analytical guard cartridge system $(4.6 \times 10 \mathrm{~mm})$, cartridge guard column, and $\mathrm{C}_{18}$ cartridge security guard $(4 \times 3 \mathrm{~mm})$.

\subsection{Milk analysis}

Milk samples were analyzed at the start and at the end of the trial for fat, protein, and lactose with a Milkoscan 605 (Foss Electric, Hillerød, Denmark), for somatic cell count (SCC) with a Fossomatic 360 (Foss Electric) and for total bacterial count (TBC) with a Bactoscan 8000 (Foss Electric).

\subsection{Blood analysis}

Blood samples were collected from each donkey by jugular venepuncture before and after the trial for immediate analysis. Hematocrit (HCT) was determined with a Baker System instrument (9120 AX, Biochem Immunosystems Italy).

Serum samples were analyzed (Photometer 4010 Boehringer Mannheim, Germany) with colorimetric commercial kits (Roche Diagnostics, Mannheim, Germany) for total creatinine, bilirubin, aspartate amine transaminase (AST), alanine amine transaminase (ALT), glutamic gamma transaminase (GGT), lactate dehydrogenase (LDH).

\subsection{Carryover calculation}

The carryover was calculated as individual daily ratio between the AFM1 excreted in milk and the corresponding AFB1 intake, during the time in which the steady-state output of toxin in milk was reached. The carryover of AFM2 after AFB2 intake was also performed.

Following as reported by Littell et al. (1998), the steady-state period for each aflatoxin was established as starting from the first hour of sampling that was not significantly different $(P>0.10)$ from average of subsequent hours, within the time of administration of CNC. 


\subsection{Statistical analyses}

The milk and blood parameters and the AFM1, AFM2 content in milk (to establish the beginning and the duration of the period of steady-state output of these aflatoxins in milk) were performed by repeated measure analysis (ANOVA) test using JMP ${ }^{\circledR}$ (19892007, Version 7, SAS Institute Inc., Cary, NC).

\section{Results and discussion}

None of the donkeys employed showed clear adverse effects on health during the experimental period.

All animals ingested the total administered ration daily. At the end of the trial, the donkey body weight was $260.16 \pm 7.34 \mathrm{~kg}$ (mean \pm SD). The BW was not negatively affected by the presence of AFs in the diet and remained constant over the observation period.

The milk composition as well as SCC and TBC content are reported in Table 1. Fat, protein, and lactose concentrations did not significantly vary with AFs ingestion. The SCC content was not influenced by the intake of AFs, as previously observed in cows (Applebaum et al. 1982), and was comparable with the normal values reported in lactating donkeys by Colavita et al. (2011). The total bacterial cell count was also under the threshold of 1,500,000 per mL (Reg. EC 853/2004).

The serum and the hematological parameters measured at the beginning and at the end of the trial are reported in Tables 2 and 3, respectively. Statistical analysis underlined that the aflatoxin intake did not significantly affect the serum parameters related to liver function. As serum enzyme activities are generally elevated in aflatoxicosis as a consequence of hepatocyte damage (Pier 1992), this result suggests that administration of AFB1 and AFB2 by feeding in this trial did not cause significant hepatic damage. No effects due to aflatoxin were observed for any of the hematological parameters considered and they were within the normal range.

No AFM1 and AFM2 were detected in the milk before the CNC administration. AFM1 was detected in milk of all animals beginning from $1 \mathrm{~h}$ after the first CNC administration (Fig. 1) in agreement with a previous study in goats (Mazzette et al. 2009). In dairy cows, Moschini et al. (2007) detected AFB1 and AFM1 in plasma as early as $15 \mathrm{~min}$ after the ingestion of feed contaminated by aflatoxins. These results

Table 1 Donkeys milk $(n=6)$ characteristics before and after the feeding with naturally contaminated corn

\begin{tabular}{lllll}
\hline & Time 0 & End of the trial & SEM & $P$ value \\
\hline Milk yield (L) & 2.03 & 1.95 & 0.04 & 0.18 \\
Fat $(\%)$ & 0.10 & 0.11 & 0.10 & 0.10 \\
Protein $(\%)$ & 1.59 & 1.57 & 0.01 & 0.13 \\
Lactose $(\%)$ & 6.31 & 6.19 & 0.09 & 0.39 \\
SCC $\left(\times 10^{3} \cdot \mathrm{mL}^{-1}\right)$ & 27.80 & 26.97 & 0.42 & 0.22 \\
TBC $\left(\times 10^{4} \mathrm{CFU} \cdot \mathrm{mL}^{-1}\right)$ & 3.43 & 3.60 & 0.11 & 0.33 \\
\hline
\end{tabular}

SCC Somatic cell count TBC Total bacterial count $C F U$ colony forming unit 
Table 2 Average of serum parameters $(n=6)$ of donkeys before and after the feeding with naturally contaminated corn

\begin{tabular}{lllll}
\hline & Time 0 & End of the trial & SEM & $P$ value \\
\hline GGT (I.U.) & 21.67 & 21.00 & 1.32 & 0.74 \\
AST (I.U.) & 312.83 & 313.17 & 0.89 & 0.80 \\
ALT (I.U.) & 18.00 & 17.00 & 2.19 & 0.76 \\
LDH (I.U.) & 416.00 & 418.33 & 4.47 & 0.73 \\
Creatinine (mg.dL & & 1.07 & 0.08 & 0.15 \\
Total bilirubin, $\left(\mathrm{mg}^{-1} \mathrm{dL}^{-1}\right)$ & 1.26 & 0.12 & 0.02 & 0.30 \\
\hline
\end{tabular}

GGT glutamic gamma transaminase, $A S T$ aspartate amine transaminase, $A L T$ alanine amine transaminase, $L D H$ lactate dehydrogenase

suggest that the absorption of AFB1 in the gastrointestinal tract and its liver oxidation are very quick processes.

AFM2 was also detected in donkey milk at the first milking $(1 \mathrm{~h})$ from the first ingestion of AFB2-contaminated feed (Fig. 1).

During the CNC administration period, in all animals, AFM1 and AFM2 content increased in milk from 0 to $104 \mathrm{~h}\left(32.25 \mathrm{ng} . \mathrm{L}^{-1}\right)$ and to $128 \mathrm{~h}\left(23.33 \mathrm{ng} . \mathrm{L}^{-1}\right)$, respectively (Fig. 1). Then, AFM1 and AFM2 contents decreased and reached a steady-state condition in the interval between 152 and 296 h. A similar trend of AFM1 has also been found by Battacone et al. (2003) on sheep, by Ronchi et al. (2005) on lactating goats, and by Bertuzzi et al. (2003) on lactating sows. The highest value of AFM1 concentration in milk (32.25 ng. $\mathrm{L}^{-1}$ ) was below the European Community maximum allowed in AFM1 milk levels for adult consumption (50 ng. $\mathrm{L}^{-1}$ ) but above the AFM1 milk levels allowed for infants $\left(25 \mathrm{ng} . \mathrm{L}^{-1}\right)$.

The AFM2 concentration at 32, 56, 104, and $128 \mathrm{~h}$ was more than $20 \mathrm{ng} . \mathrm{L}^{-1}$. These values proved to be similar to those found for AFM1, although the AFB2 in the $\mathrm{CNC}$ administered was 18 times less than the AFB1 (11 vs $202 \mu \mathrm{g} \cdot \mathrm{kg}^{-1}$ ). Also,

Table 3 Average of hematological parameters $(n=6)$ of donkeys before and after the feeding with naturally contaminated corn

\begin{tabular}{lllll}
\hline & Time 0 & End of trial & SEM & $P$ value \\
\hline $\mathrm{WBC} \times 10^{3}$ cell. $\mu \mathrm{L}^{-1}$ & 10.77 & 11.43 & 0.22 & 0.09 \\
$\mathrm{RBC} \times 10^{6}$ cell. $\mu \mathrm{L}^{-1}$ & 7.70 & 7.75 & 0.11 & 0.76 \\
$\mathrm{HGB}\left(\mathrm{g} . \mathrm{dL}^{-1}\right)$ & 11.78 & 13.06 & 0.49 & 0.12 \\
$\mathrm{HCT}(\%)$ & 35.83 & 35.43 & 0.66 & 0.68 \\
$\mathrm{MCHC}\left(\mathrm{g} . \mathrm{dL}^{-1}\right)$ & 34.92 & 35.20 & 0.25 & 0.45 \\
$\mathrm{MCH}(\mathrm{pg})$ & 16.02 & 15.63 & 0.35 & 0.47 \\
$\mathrm{MCV}(\mathrm{fL})$ & 44.75 & 45.07 & 0.57 & 0.71 \\
\hline
\end{tabular}

$W B C$ white blood cell count, $R B C$ red blood cell count, $H G B$ hemoglobin, $H C T$ hematocrit, $M C H C$ mean corpuscular hemoglobin concentration, $M C H$ mean corpuscular hemoglobin, $M C V$ mean corpuscular volume 


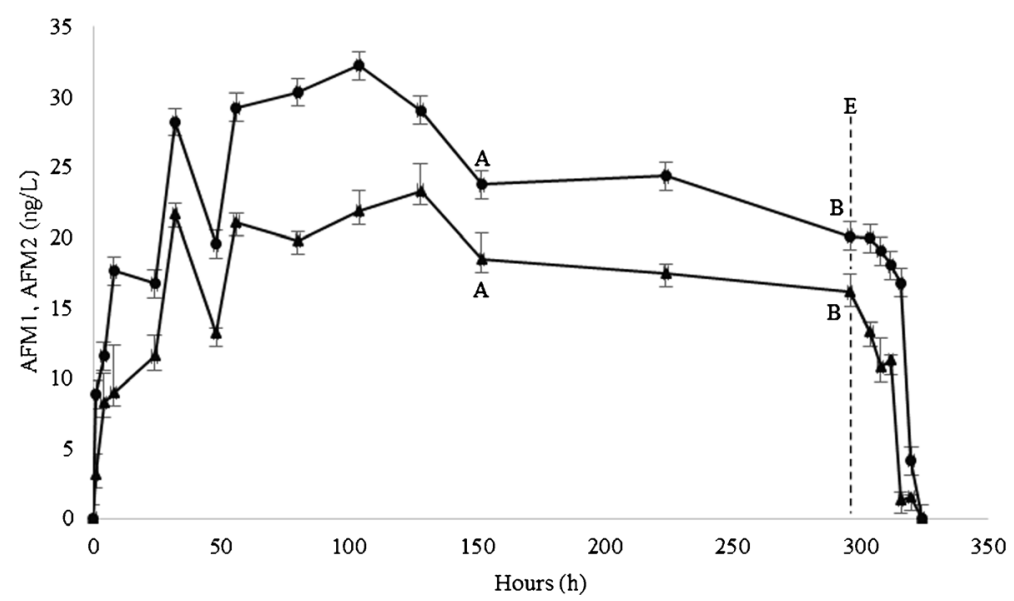

Fig. 1 Aflatoxin M1 (AFM1) (black circle) and aflatoxin M2 (AFM2) (black triangle) concentrations (mean \pm S.E. $)$ in milk of donkeys $(n=6)$. $A-B$ : Steady-state period, where $A$ was the first hour of sampling that did not differ significantly $(P>0.10)$ from the next up to $B$ hour. E (dotted line): end time of administration of contaminated corn

other authors have reported a higher passage of AFM2 in milk of sows and buffalos (Bertuzzi et al. 2003; Pietri et al. 2003).

In the clearance period, AFM1 and AFM2 quickly decreased, and they were no longer detected at $28 \mathrm{~h}$ after the end of CNC administration (Fig. 1). Similar results were found in sows (Bertuzzi et al. 2003). The total disappearance of AFM1 from the last AFB1 administration seems to be faster in monogastric species than ruminants. In fact, in dairy cows and in small ruminants, disappearance time was about 3-4 days from the last AF administration (Ronchi et al. 2005; Battacone et al. 2003; Masoero et al. 2007).

The similar AFM1 and AFM2 trend and the disappearance rate in milk are probably related to the similar molecular structure. Pietri et al. (2003) and Bertuzzi et al. (2003) carried out trials on buffalos and sows and found that AFM1 and AFM2 disappeared from the milk at the same time.

The AFM1 and AFM2 carryover into milk, observed during the steady state, proved to be $0.02 \% \pm 0.005$ and $0.31 \% \pm 0.020$ (means $\pm \mathrm{SE}$ ), respectively. This carryover AFM1 value was lower compared with that found in other species, which ranged from $0.06 \%$ in sow, $0.2 \%$ in buffalo, $1.8 \%$ in goats, $2.90 \%$ in sheep to $6.6 \%$ in cows (Bertuzzi et al. 2003; Pietri et al. 2003; Ronchi et al. 2005; Battacone et al. 2009; Britzi et al. 2013). The AFM1 carryover appears to be clearly influenced by the animal species; in particular, the carryover in monogastric animals seems to be lower than that found in ruminants. Ruminants are generally considered more resistant than monogastric animals, due to the adverse effects of mycotoxins, because of the rumen and its microbial population should play a role in detoxification. Ruminal and hepatic bioconversions of mycotoxins alter the polarity of toxins and their affinity to water or lipids, consequently ruminants excretion of toxins and their metabolites in milk results higher than monogastrics (Yannikouris and Jouany 2002; Jouany et al. 2009).

The carryover of AFM2 was about 15 times higher than that found with AFM1. This result was in agreement with previous trials in buffaloes (Pietri et al. 2003) and in sows (Bertuzzi et al. 2003). 
The higher rate of passage in milk of AFM2 might be related to the lower amount of AFB2 present in contaminated feed. An inverse correlation was observed between AFB1 administered by feeding and AFM1 excreted in sheep and goats milk (Battacone et al. 2009; Ronchi et al. 2005; Nageswara Rao and Chopra 2001).

The aflatoxin M2 result suggests that careful consideration should be given to the presence of this aflatoxin, which is not regulated by the law in all species of dairy mammals. The level of toxicity of AFM2 could be less than that of AFM1. This may be due to the lack of the double bond terminating the difuran ring system which is common in AFB1, AFG1, and AFM1 (Weindenbörner 2001). In this regard, there are, however, few references demonstrating lower AFM2 toxicity in infants than that of AFM1. Following the precautionary principle, we feel entitled to consider the likely effects of the sum of these two aflatoxins. In our trial, the sum of the AFM1 and AFM2 concentrations exceeded the legal limit for the content of aflatoxin M1 in baby food and infant formula $\left(25 \mathrm{ng} . \mathrm{L}^{-1}\right)$, as well as the legal limit for the content of AFM1 in milk (50 ng. $\left.\mathrm{L}^{-1}\right)$ (Reg. EC 1881/2006). Bianco et al. (2012) highlighted that the contemporary presence of mycotoxins AFB1, AFB2, and their respective metabolites AFM1 and AFM2 in foods, could significantly contribute to altering immune response against tumor cells, resulting in a reduction of life span and an increase of carcinogenesis.

Low levels of AFB1 (1-5 ng. $\left.\mathrm{L}^{-1}\right)$ were also found in six samples of milk. This very low excretion of unmetabolized AFB1 was insufficient to estimate the rate of passage in donkey milk. Other authors have reported a rate of passage of AFB1 in milk $(0.45 \%$ buffalo milk, Pietri et al. 2003; 0.07\% sow milk, Bertuzzi et al. 2003). Even in breast milk, a remarkable concentration of AFB1 was found (Gürbay et al. 2010).

\section{Conclusion}

This trial showed that the presence of aflatoxins B1 and B2 in feed causes a carryover in donkey milk. The carryover observed on aflatoxin M2 was higher than on M1 and was 0.31 and $0.02 \%$, respectively. AFM1 carryover was lower than that reported in other species, while AFM2 showed a rate of passage comparable with some data in the literature. The direct transfer of AFB1 in milk was detected, although at very low amounts.

This work on the transfer of aflatoxins from contaminated feed to donkey milk is a first approach to the problem. Further studies and investigations will be useful in order to evaluate the possible effect of other variables such as the different stages of lactation and the dose of aflatoxins ingested. Nonetheless, the aflatoxins content in donkey milk should be taken into serious consideration.

\section{Compliance with ethical standards}

Conflict of interest Author Tozzi, author Liponi, author Meucci, author Casini, author Dall'Asta, author Intorre and author Gatta declare that they have no conflict of interest.

Statement of animal rights All applicable institutional and national guidelines for the care and use of laboratory animals were followed. 


\section{References}

Applebaum RS, Brackett RE, Wiseman DW, Marth EH (1982) Response of dairy cows to dietary aflatoxin: feed intake and yield, toxin content, and quality milk of cows treated with pure and impure aflatoxin. J Dairy Sci 65:1503-1508. doi:10.3168/jds.S0022, 0302(82)82374-6

Ardic M, Karakaya Y, Atasever M, Durmaz H (2008) Determination of aflatoxin B1 levels in deep red ground pepper (isot) using immunoaffinity column combined with ELISA. Food Chem Toxicol 46(5):15961599. doi:10.1016/j.fct.2007.12.025

Battacone G, Nudda A, Cannas A, Cappio Borlino A, Bomboi G, Pulina G (2003) Excretion of aflatoxin M1 in milk of dairy ewes treated with different doses of aflatoxin B1. J Dairy Sci 86:2667. doi:10.3168/jds. S0022-0302(03)73862-4

Battacone G, Nudda A, Palomba M, Mazzette A, Pulina G (2009) The transfer of aflatoxin M1 in milk of ewes fed diet naturally contaminated by aflatoxins and effect of inclusion of dried yeast culture in the diet. J Dairy Sci 92:4997-5004. doi:10.3168/jds.2008-1684

Bertuzzi T, Pietri A, Barbieri G, Piva G (2003) Aflatoxin residues in milk of sows fed a naturally contaminated diet. Ital J Anim Sci 2(suppl1):234-236. doi:10.4081/ijas.2003.s1.234

Biancardi A, Piro R, Dall'Asta C, Galaverna G (2013) A simple and reliable liquid chromatography-tandem mass spectrometry method for the determination of aflatoxin M1 in milk. Food Addit Contam 30(2):381388. doi:10.1080/19440049.2012.743041

Bianco G, Russo R, Marzocco S, Velotto S, Autore G, Severino L (2012) Modulation of macrophage activity by aflatoxins B1 and B2 and their metabolites aflatoxins M1 and M2. Toxicon 59:644-650. doi:10.1016/j. toxicon.2012.02.010

Bilandzic N, Bozic D, Dokic M, Sedak M, Kolanovic BS, Varenina I, Cvetnic Z (2014) Assessment of aflatoxin M1 contamination in the milk of four dairy species in Croatia. Food Control 43:18-21. doi:10. 1016/j.foodcont.2014.02.0440956-7135

Britzi M, Friedman S, Miron J, Solomon R, Cuneah O, Shimshoni JA, Soback S, Ashkenazi R, Shlosberg A (2013) Carry-over of aflatoxin B1 to aflatoxin M1 in high yielding Israeli cows in mid- and late-lactation. Toxins 5:173-183. doi:10.3390/toxins5010173

Colavita G, Amadoro C, Salimei E (2011) Sicurezza alimentare. Latte d'asina: aspetti igienico- sanitari e normativi. Argomenti 3(XIII):61-70

Decastelli L, Lai J, Gramaglia M, Monaco A, Nachtmann C, Oldano F, Ruffier M, Sezian A, Bandirola C (2007) Aflatoxins occurrence in milk and feed in Northern Italy during 2004-2005. Food Control 18(10): 1263-1266. doi:10.1016/j.foodcont.2006.08.006

Faye B, Konuspayeva G (2012) The sustainability challenge to the dairy sector e the growing importance of non-cattle milk production worldwide. Int Dairy J 24:50-56. doi:10.1016/j.idairyj.2011.12.011

Fink-Gremmels J (2008) Mycotoxins in cattle feeds and carry-over to dairy milk: a review. Food Addit Cont 25:172-180. doi:10.1080/02652030701823142

Gürbay A, Atasayar Sabuncuoglu S, Girgin G, Șahin G, Yiğit Ș, Yurdakök M, Tekinalp G (2010) Exposure of newborns to aflatoxin M1 and B1 from mothers' breast milk in Ankara, Turkey. Food Chem Toxicol 48: 314-319. doi:10.1016/j.fct.2009.10.016

Hernández-Martínez R, Navarro-Blasco I (2010) Aflatoxin levels and exposure assessment of Spanish infant cereals. Food Addit Contam Part B Surveill 3(4):275-288. doi:10.1080/19393210.2010.531402

Herzallah S (2009) Determination of aflatoxins in eggs, milk, meat and meat products using HPLC fluorescent and UV detectors. Food Chem 114(3):1141-1146. doi:10.1016/j.foodchem.2008.10.077

IARC (2002) IARC Monographs on the evaluation of carcinogenic risk to human, vol 82, Some traditional herbal medicines, some mycotoxins, naphthalene and styrene. International Agency for Research on Cancer, Lione

Jirillo F, Jirillo E, Magrone T (2010) Donkey's and goat's milk consumption and benefit to human health with special reference to the infiammatory status. Curr Pharm Des 16:859-863

Jouany JP, Yannikouris A, Bertin G (2009) Risk assessment of mycotoxins in ruminants and ruminant products. Options Méditérr 85:205-224

Kos J, Levic J, Duragic O, Kokic B, Miladinovic I (2013) Occurrence and estimation of aflatoxin M1 exposure in milk in Serbia. Food Control 38:41-46. doi:10.1016/j.foodcont.2013.09.060

Littell RC, Henry PR, Ammerman CB (1998) Statistical analisis of repeated measures data using SAS procedures. J Anim Sci 76:1216-1231

Lopez CE, Ramos LL, Ramadan SS, Bulacio LC (2003) Presence of aflatoxin M1 in milk for human consumption. Argentina Food Control 14:31-34 
Masoero F, Gallo A, Moschini M, Piva G, Diaz D (2007) Carryover of aflatoxin from feed to milk in dairy cows with low or high somatic cell counts. Anim Res 1:1344-1350. doi:10.1017/S1751731107000663

Mazzette A, Decandia M, Acciaro M, Fenu A, Francesconi AHD, Battacone G (2009) Excretion of aflatoxin M1 in milk of goats fed diet contaminated by aflatoxin B1. Ital J Anim Sci 8(2):631-633. doi:10.4081/ijas.2009.s2.631

Meucci V, Razzuoli E, Soldani G, Massart F (2010) Mycotoxin detection in infant formula milks in Italy. Food Addit Contam 27(1):64-71. doi:10.1080/02652030903207201

Moruzzo R, Rossignoli C (2013) Nicchia ad alto valore commerciale. Largo consumo, No.12,31

Moschini M, Masoero F, Gallo A, Diaz D (2007) Mucosal absorption of aflatoxin B1 in lactating dairy cows. Ital J Anim Sci 6(suppl1):324-326. doi:10.4081/ijas.2007.1s.324

Nageswara Rao SB, Chopra RC (2001) Influence of sodium bentonite and activated charcoal on aflatoxin M1 excretion in milk of goats. Small Rum Res 41:203-213

Pier AC (1992) Major biological consequences of aflatoxicosis in animal production. J Anim Sci 70:3964-3967

Pietri A, Bertuzzi T, Fortunati P, Gualla A (2003) Excretion pattern of aflatoxins in buffalo milk and carry-over in mozzarella cheese. Ital J Anim Sci 2(suppl1):302-304

Ronchi B, Danieli PP, Vitali A, Sabatini A, Bernabucci U, Nardone A (2005) Evaluation of AFB1/AFM1 carry-over in lactating goats exposed to different levels of AFB1 contamination. 56th Annual Meeting of the EAAP, Uppsala, Sweden, June 2005

Weidenbörner M (2001) Enciclopedia of food mycotoxins. Springer-Verlag Berlin Heidelberg, New York, p 10

Yiannikouris A, Jouany JP (2002) Mycotoxins in feeds and their fate in animals: a review. Anim Res 51:8199. doi:10.1051/animres:2002012 